\title{
L'ARGOT DE LA GUERRE DE SARAJEVO, UN EXEMPLE DU RAPPORT DE LA LANGUE, DE SON LEXIQUE AU VÉCU
}

\section{INTRODUCTION : RAPPORTS ARGOT - GUERRE}

L'argot repose sur la différence entre nous et les autres, ceux qui sont différents et que nous excluons de la communication. Nous pouvons ainsi donner la formule minimale de l'argot de façon suivante : (1+1)-1. La différence en question est le plus souvent sociale mais peut être aussi : géographique, ethnique, religieuse, nationale, etc. La guerre est une situation « argotisante » par excellence parce qu'elle implique la partiton entre nous et les autres, qui sont nos ennemis. Du point de vue des civils de Sarajevo pendant la guerre en Bosnie-Herzégovine, ces « autres » étaient multiples et la perception des Sarajeviens allait de la haine à la désillusion. Ainsi, on peut distinguer:

1. ennemis directs (Serbes agresseurs dénommés tchetniks ${ }^{l}$ et à différencier des Serbes restés à Sarajevo, solidaires des Bosniaques et des Croates);

2. ceux qui ont abandonné et délaissé les Sarajeviens:

a) en fuyant la ville et/ou le pays, comparables à des lâches et des traîtres ${ }^{2}$;

b) les Casques Bleus qui ne protégeaient guère les habitants et dont le rôle se réduisait au maintien du statu quo et à l'acheminement de l'aide humanitaire ;

c) la communauté internationale qui interprétait mal le conflit et n'intervenait pas.

La perception du vécu des côtés opposés, dans cette guerre, varie extrêmement, encore aujourd'hui. Selon Erving Goffman (Goffman 1974: 18), les descriptions rétrospectives d'un " même " événement ou d'une " même » circonstance sociale peuvent diverger considérablement et le rôle de chacun dans une activité le conduit à se faire sa propre idée de ce type d'activité. Ainsi, par exemple, les supporters de deux équipes de football ne vivent pas le même match. Cette situation pourrait être transposée à une situation de guerre où les différents côtés, qui y participent, ont tous leur propre perception et interprétation des choses. Sauf qu'après cette guerre-ci, ces interprétations différentes continuent et font penser au film Rashômon de Kurosawa. Parce que, encore à ce jour, en Bosnie-Herzégovine, nous avons trois versions des

*alma.sokolija@gmail.com

1 Tchetnik (četnik < četa, ,,compagnie en armée“) fait référence à des unités militaires serbes de la Deuxième guerre mondiale qui ont participé dans des massacres sur des Bosniaques et des Croates.

2 D'où les argotismes comme smrad et pobljegulja; à voir plus loin dans le texte. 
faits, avec trois interprétations différentes de l'histoire, trois partis politiques nationaux au pouvoir et trois présidents à la tête de la Présidence. C'est une situation des plus surréalistes, mais elle existe pour satisfaire les majorités nationales, en respectant, ce qu'on appellait à l'époque du communisme le „clef national“ (nacionalni $k l j u c$ c). Celui-ci exige une représentativité égale de tous les peuples majoritaires dans toutes les institutions du pouvoir (i.e. des Bosniaques qui s'appellaient alors des $\mathrm{Mu}-$ sulmans ${ }^{3}$, des Croates et des Serbes).

\section{QUELQUES DONNÉES SUR LE SIÈGE DE SARAJEVO}

Le siège de Sarajevo a duré 44 mois (depuis 5 Avril 1992 jusqu'au 29 Février 1996). On considère que c'est un des plus longs sièges de ville dans l'histoire des guerres. On dénombre 11541 morts dont 1601 enfants et environ 50000 blessés. La ville a été bombardée en permanence, en moyenne par 330 obus par jour. On note aussi des journées, comme le 22 juillet 1993, où 3777 obus s'abattent sur la ville et ses habitants. La population survit tant bien que mal : la nourriture se réduit dans la plupart des cas à l'aide humanitaire acheminée par les Casques Bleus. Cela explique le mépris des Sarajéviens pour les Casques Bleus : ils ne protègent guère les populations. Ils leur permettent juste de survivre très péniblement en maintenant cette situation à l'infini. D'autre part le marché noir fleurit. Le prix des cigarettes, de l'alcool ou des produits alimentaires, très simples, dépassent, de loin, ceux des pays libres d'Europe, à l'époque. Grâce à ce marché noir, certains vivent très bien tandis que les autres meurent de faim. Il est aussi très difficile de tenir sans eau car les Serbes agresseurs encerclent la ville et coupent l'eau. Les habitants sont obligés de se rendre à des fontaines publiques, en risquant leur vie, pour s'approvisionner en eau quotidiennement. L'agresseur guette souvent ces rassemblements, pour y lancer des obus et provoquer des massacres. La ville est aussi privée d'électricité et de gaz, ce qui est surtout mal vécu en hiver. En effet, les hivers peuvent y être rudes et les températures descendre jusqu'à moins vingt degrés. De plus, toutes les vitres des fenêtres sont brisées à cause des détonations et elles sont remplacées par du nylon. La ville est située à 550 mètres d'altitude dans une cuvette qui s'ouvre d'un seul côté vers la plaine. La visibilité est parfaite pour les agresseurs qui sont situés sur les collines.

L'aéroport, situé dans cette plaine, est tenu par les Casques Bleues, qui permettent le ravitaillement en aide humanitaire. Pour mieux décrire Sarajevo pendant la guerre, il est nécessaire de parler aussi des roses de Sarajevo, du tunnel de Sarajevo et de l'allée des snipers.

\subsection{Les roses de Sarajevo}

Les roses de Sarajevo sont les traces d'obus qui explosent sur le bitume. Elle créent des cratères aux nombreux éclats qui évoquent des pétales de fleur. Au sortir de la guerre en 1996, des habitants ont rempli plusieurs de ces impacts de résine rouge. Ces roses rouge sang, souvenirs de la guerre, jalonnent les trottoirs et la chaussée. Certaines ont disparues, d'autres sont restées.

3 Les Musulmans constituaient alors une nationalité d'où la lettre majuscule. 


\subsection{Le tunnel de Sarajevo}

En 1993, alors que les forces Serbes vont, semble-t-il, parvenir à achever l'encerclement de la ville au mont Igman, un tunnel passant sous la piste de l'aéroport de Sarajevo, alors zone neutre sous contrôle de l'ONU, permet aux forces bosniaques de contenir l'avancée serbe sur ce même mont et à de nombreux civils de quitter la ville assiégée. Il reliait le centre de Sarajevo à Butimir, petite localité au pied du Mont Igman. Dans son récit de voyage en Bosnie-Herzégovine, le linguiste français Paul Garde raconte comment il doit l'emprunter pour entrer à Sarajevo en octobre 1994 :

Je prends le sac, je sors, personne ne m'arrête. Je suis dans le tunnel. C'est un boyau étroit, comme une galerie de mine (ce sont, me dira-t-on, les mineurs de Zenica qui l'ont creusé). Il est éclairé faiblement, mais suffisamment. On peut y croiser ou y dépasser à la rigueur, mais en se serrant bien contre la paroi, comme dans un couloir de train. Normalement on ne croise pas. Le tunnel, me dira-t-on, fonctionne une demi-heure dans chaque sens. ${ }^{4}$

Paul Garde fut un des rares étrangers à franchir ce tunnel, qui servit jusqu'à la levée du siège en 1995, et il raconte aussi comment on l'a accueilli alors à l'Ambassade de France à Sarajevo :

Le conseiller m'interroge sur mon voyage et paraît stupéfait d'apprendre que j'ai emprunté le tunnel. A l'ambassade, on n'a, paraît-il jamais rencontré personne qui y soit passé. Il semble qu'il soit réservé aux Bosniens et je suis, à sa connaissance, le seul étranger qui l'ait traversé. [...] Chose curieuse, les gens d'ici parlent de passer ce tunnel comme s'il s'agissait de prendre le métro ; les étrangers, eux, me regardent comme si j'avais gravi l'Annapurna. Rien ne montre mieux le gouffre existant entre les deux mondes. ${ }^{5}$

Cet écart, ce décallage énorme, entre le statut des Sarajeviens en pleine guerre et le statut de tous les autres (des étrangers, des Casques Bleues et des diplomates qui vivaient alors dans la ville) était tout à fait exaspérant pour les Sarajeviens. Alors qu'ils mouraient de faim et devaient passer par le tunnel pour sortir de la ville, les autres étaient protégés, bien nourris, souvent cyniques. Ils se sentaient apartenir à un autre monde.

Les assaillants serbes tentèrent d'anéantir le tunnel à plusieurs reprises. Soit en bombardant ses accès, soit en tentant de l'inonder en creusant un tunnel adjacent, relié à la nappe phréatique. Sans ce tunnel, jamais les hommes, les vivres, les armes et les munitions nécessaires à la défense de la ville et à la survie de la population, n'auraient pu y entrer. Les forces de l'ONU ne prirent jamais l'initiative de le détruire, probablement par crainte des éventuelles réactions de la communauté internationale que cet acte aurait pu entraîner.

4 Le siège de Sarajevo sur Wikipedia: https://fr.wikipedia.org/wiki/Si\%C3\%A8ge_de_Sarajevo

5 Ibid. 


\subsection{Allée de snipers}

Elle désignait l'avenue principale de Sarajevo lors de son siège par l'Armée de la République serbe de Bosnie, pendant la guerre de Bosnie-Herzégovine. Ceci en raison du nombre de snipers qui la prenaient pour cible. L'une des rares sources d'eau potable de la ville se trouvait sur cette avenue qui relie la zone industrielle au centre historique de la ville. L'avenue est bordée de hauts bâtiments donnant aux snipers de nombreuses positions de tir. Les montagnes autour de la ville offraient également aux snipers une distance de sécurité, doublée d'une bonne visibilité sur la ville et son trafic. Bien que constamment sous le siège de l'armée serbe, la vie normale continuait, des panneaux indiquant aux civils la présence de snipers (Pazi snajper!="Fais attention, sniper!"). Selon les données récoltées en 1995, ceux-ci ont blessé 1030 personnes et en ont tué 225 , dont 60 enfants lors du siège de la ville.

\section{4. Fin de la guerre}

En 1995, les forces internationales se retournèrent fermement contre les attaquants, notamment lors du combat du pont de Vrbanja à la suite de la prise en otage de plusieurs centaines d'observateurs de l'ONU. Les forces serbes pillèrent aussi un point de collecte d'armes des Nations Unies, ce qui entraîna la décision de l'OTAN d'autoriser le bombardement aérien des dépôts de munitions serbes. Les combats se poursuivirent et les Serbes perdirent progressivement du terrain. Le chauffage, l'électricité et l'eau furent rétablis en ville. Un cessez-le-feu fut décrété en octobre 1995, et les accords de Dayton furent ratifiés à la fin de l'année, apportant la fin des hostilités, la stabilité et un retour à la normale. Le gouvernement de la République de Bosnie-Herzégovine déclara officiellement la fin du siège de Sarajevo le 29 février 1996. La Bosnie-Herzégovine d'aujourd'hui est constituée de deux entités (La Fédération de Bosnie-Herzégovine, la République serbe) et d'un district (Brčko).

La Fédération de Bosnie-Herzégovine est majoritairement peuplée de Bosniaques (musulmans) et de Croates (catholiques) et la République serbe est majoritairement peuplée de Serbes (orthodoxes). En dehors de ces religions majoritaires, il reste en Bosnie-Herzégovine aussi un certains nombre de juifs, d'ashkénazes aussi bien que de sépharades. Cette mixité religieuse qui en temps de paix fait la richesse du pays et son cosmopolitisme, en temps de guerre fait sa faiblesse et son caractère de poudrière des Balkans. ${ }^{6}$

La paix conclue en quelque sorte enterine la purrification éthnique et la tendance vers une ultérieure purification des territoires continue avec les migrations de populations.

6 Un dicton populaire dit I mirna Bosna... («Et que la Bosnie soit en paix... »), ce qui veut dire et « et qu'on en finisse avec les conflits (verbaux)»; « et qu'on se réconcilie (même difficilement) ». Il fait référence à cette Histoire souvent sanglante du pays. Certains historiens mentionnent surtout le Moyen âge où les guerres en Bosnie éclataient très souvent de sorte que le règne de Kulin Ban était considéré comme le bon vieux temps parce que à cette époque il y a eu une période de paix de 25 ans. Il faut ajouter que la Bosnie a tenu un « record» sous Tito avec 46 années de paix. 


\subsection{La composition ethnique et religieuse de la Bosnie-Herzégovine}

Pour comprendre la composition ethnique et religieuse de la Bosnie-Herzégovine d'aujourd'hui ou même d'avant cette dernière guerre, il est nécessaire d'en donner un bref aperçu historique. Avant l'arrivée des peuples slaves sur les Balkans, la région a été habitée par des tribus illyriennes, celtes et thraces. L'Empire Romain amène le christianisme. Avec la partition de celui-ci, les Balkans sont coupés en deux: à l'ouest fleurit le catholicisme et à l'est l'orthodoxie. Ce partage entraîne la polaristion entre les Croates (catholiques) et les Serbes (orthodoxes). Cette frontière passe par l'actuelle Bosnie-Herzégovine.

Au Moyen âge sous le roi Tvrko Kotromanjić, la Bosnie connait une apogée et ses territoires s'élargissent vers la mer tandis que sur le plan religieux l'église cathare, dualiste, devient la religion officielle de l'État ${ }^{7}$. Avec l'arrivée des Ottomans les frontières de Bosnie se redessinent et une nouvelle religion (l'islam) fait son apparition. Cette colonisation va durer cinq siècles en Bosnie. Et finalement avec l'arrivée des AustoHogrois les frontières bougent encore.

\section{L'ARGOT DES CIVILS DANS LA GUERRE OU L'INVERSION DES RÉALITÉS}

L'argot de la guerre de Sarajevo est caractérisé par une inversion des réalités : alors que dans la ville, qui subit l'agression, domine une certaine intensification de l'emploi de termes argotiques car on utilise des termes de la guerre pour désigner les choses communes, au front, qui est tout près, on utilise des euphémismes pour atténuer la réalité trop brutale. Ainsi quand on parle des « cigarettes » on dit " munitions » (municija). Pour dénommer une " grande bouteille de bière » on dit " obus » (granata). Pour dire « paquet de cigarettes » on dit «VBR». Le VBR est un sigle de višecijevni bacač raketa (« lanceur multicanonique des obus»). En fait, à l'époque de la plus grande pénurie de cigarettes et de papier d'emballage, on enveloppait les cigarettes dans des pages déchirées de livres. Vues de côté, vingt cigarettes rappelaient les tuyaux d'un VBR.

Probablement parce que la ville a été constamment sous les obus qu'on "lance » (ispaliti), on crée un nouveau terme « se faire lancer» (ispaliti se) qui désigne le mouvement inverse et veut dire qu'on " est sorti de Sarajevo encerclé ». Ou alors, il peut désigner le mouvement dans le sens plus général car on pouvait « se faire lancer » jusqu'au café du coin, c'est-à-dire, " aller (quelque part) ». Aujourd'hui ce terme s'est maintenu dans le sens « partir de Bosnie-Herzégovine pour aller vivre à l'étranger », ce qui témoigne d'une part du sentiment d'enfermement et de la fragilité de cette paix et d'autre part de la situation économique catastrophique du pays. Un deuxième terme qui s'est maintenu jusqu'à aujourd'hui est pući (« exploser ») pour dire « disjoncter », « sombrer dans la folie ». D'un côté c'est parce que pendant la guerre les civils étaient entourés d'explosions et de détonations différentes et, d'autre côté, parce que cet état, souvent transitoire, était relativement fréquent pendant la

7 Les croisades qui vont s'ensuivre vont aboutir à l'extermination les cathares. 
guerre, ce qui est tout à fait compréhensible. Alors que l'argot de la ville est caractérisé par des termes propres à la guerre, sur le front domine l'euphémisation et pour dire qu'on «a tué » ou qu'on « a détruit» on utilise le terme odratiti (" faire ») pour ainsi masquer un peu la réalité trop brutale.

\section{LES TABOUS, LES CHOSES TUES OU CHUCHOTÉES}

Chaque guerre connaît ses tabous et ses zones d'ombres. Chaque ségrégation entraîne une autre nouvelle ségrégation qui peut être tout aussi cruelle.

Ainsi l'expression pobjegulja, « fuyarde » (<pobje-ći, « fuir ») désigne :

1) les « déserteurs » aussi bien que 2) les «femmes qui ont fui la guerre (souvent avec et à cause des enfants) » ainsi que tous 3) les «Sarajevien(ne)s qui n'ont pas été à Sarajevo pendant la guerre $»^{8}$. Le terme est très stigmatisateur et on n'en parle pas encore publiquement.

Le terme podrumaš ( " cavard ») désigne un « lâche » ou celui « qui restait dans la cave au lieu d'aller sur le front » et par opposition au terme linijaš (« lignard»), celui « qui a été sur la ligne de combat ». Un peu dans le même sens, le terme maneken (« mannequin ») désignait péjorativement « celui qui se la jouait et en même temps ne participait pas à la guerre ». Il faut savoir qu'avec le temps la plupart des Sarajeviens avaient relativisé la peur de la mort et ne s'abritaient plus dans les caves, comme au début de la guerre, d'autant plus que les bombardements étaient constants.

Un des termes les plus polysémiques est certainement celui de smrad. Au départ le mot veut dire « mauvaise odeur ». Pendant la guerre il désigne les «traitres», les « mauvais», les « faux patriotes». Aujourd'hui il veut dire : 1) « con» 2) « sans caractère » 3 ) « un potin méchant qui a pour l'intention de discréditer quelqu'un », etc.

\section{CONCLUSION}

On peut se poser la question suivante : pourquoi une guerre si violente a pu produire si peu de lexèmes qui se sont maintenus jusqu'à aujourd'hui? La première hypothèse serait qu'un très grand nombre de lexèmes se sont perdus avec le temps. Les informateurs ne s'en rappellent plus ou ne veulent plus se rappeler, refoulent peut-être, exactement à cause du caractère de cette guerre. C'est peut-être encore trop douloureux car trop frais parce que la guerre est encore omniprésente aujourd'hui dans les souvenirs, les conversations, à la télévision, dans la presse et la politique. La seconde hypothèse serait qu'il s'agissait d'une guerre qu'on pourrait qualifier de guerre locale (par rapport à une guerre mondiale). Il y avait certainement des milieux où les argots spéciaux ont été créés (les unités de combat et les unités de police spéciale) mais nous avons limité notre corpus seulement à la langue des civils de Sarajevo.

Dans cet après-guerre encore douloureux, les souvenirs restent transmis et encrés dans la mémoire et l'inconscient collectif à travers ces quelques termes d'argot de la guerre de Sarajevo qui ont perduré et qui témoignent d'une réalité qui marque tout un peuple et laisse des traces dans sa langue.

$8 \quad C f$. les femmes tondues en France après la Deuxième Guerre mondiale 


\section{Bibliograhie}

GARDE, Paul (1995) Journal de voyage en Bosnie-Herzégovine, octobre 1994. Paris : La Nuée bleue.

GARDE, Paul (2000) Vie et mort de la Yougoslavie. Paris : Fayard.

GOFFMAN, Erving (1991) Les cadres de l'expérience. Paris : Les Editions de Minuit. JAHIĆ, Dževad/Senahid HALILOVIĆ/Ismail PALIĆ (éds.) (2000) Gramatika bosanskoga jezika. Zenica: Dom štampe.

MALCOLM, Noël (1996) Bosnia, a short history. New York: New York University Press. SARAČEVIĆ, Narcis (2012) Rječnik sarajevskog žargona. Sarajevo: Impresum.

SOKOLIJA-BROUILLARD, Alma (2001) Comparaison des argots de la région de Sarajevo et de la région parisienne; Approche historique, analyse linguistique et sociolinguistique des comportements et des attitudes, enquêtes et entretiens. Lille : ANRT. SOKOLIJA, Alma (2014) L'argot parisien et l'argot Sarajevien avec les dictionnaires ; Description et comparaison historiques, linguistiques et sociolinguistiques. Sarajevo: Filozofski fakultet: « fuyarde » http://www.ff-eizdavastvo.ba/Books/1\%E2\%80\%99Argot_ parisien_et_1\%E2\%80\%99argot_sarajevien_avec_les_dictionnaires.pdf

SOKOLIJA, Alma (2014) « Taboos and stigmatization and their manifestations in language and slang » In : E. Griva/D. Chostelidou (éds.), Linguistics, Culture and Identity in Foreign Language Education. Sarajevo: International Burch University Publications, 117-122.

\section{Documents consultés sur Internet}

Article sur le siège de Sarajevo sur Wikipedia. 30 octobre 2016 : https://fr.wikipedia. org/wiki/Si\%C3\%A8ge_de_Sarajevo

\section{Résumé \\ L'ARGOT DE LA GUERRE DE SARAJEVO, UN EXEMPLE DU RAPPORT DE LA LANGUE, DE SON LEXIQUE AU VÉCU}

Vingt ans après la guerre, la vie s'articule plus ou moins à Sarajevo. Les souvenirs restent, explicites ou tus. La vision de cette guerre, vécue dans l'encerclement multiple, a changé un peu. A-t-elle évolué? Il est difficile de le dire. La mémoire collective perçoit et interprète toujours certains faits comme guerriers (à tort ou à raison), certains d'autres comme des traîtrises car les plaies sont encore fraîches et ouvertes.

Les mots d'argot bosniens, utilisés dans cette guerre, possèdent leurs euphémismes, leurs tabous, leurs évitements, leurs universaux, mais ils sont aussi différents des argots de guerre dans d'autres langues parce qu'un siège de trois ans n'est pas la même chose que la guerre des tranchés. Dans cette dernière guerre en Bosnie, les « autres » étaient multiples - les ennemis autant que les traitres. Il s'agit d'une situation « argotisante » par excellence. Nous essayons de l'éclairer à travers une analyse historique du terrain. Celle-ci est nécessaire pour une analyse sémantique des lexèmes argotiques créés pendant cette guerre.

Mots clefs : argot, siège de Sarajevo, intensification, euphémisation, tabou linguistique, inversion des réalités 


\section{Abstract \\ THE WAR SLANG OF SARAJEVO, AN EXAMPLE OF RELATIONSHIP BETWEEN LANGUAGE AND ITS LEXIS TO EXPERIENCE}

Some twenty years after the war, life goes on in Sarajevo in a quasi-normal way. People are left with memories, explicit or silent. The perception of the war has not changed much. Has it evolved? It is hard to say. Rightly or wrongly the collective memory perceives and interprets certain events as acts of war and some others as treason, for the wounds are still fresh and open.

The Bosnian war slang comprises euphemisms, taboos, circumlocutions and set phrases which are in many ways different from war slang in other languages, because a three-year siege is not the same as a war of the trenches. In the war in Bosnia the "others" were multiple - both enemies and traitors. This situation has generated a large number of slang expressions, which this paper tries to present on the basis of a historical field study.

Keywords: slang, siege of Sarajevo, intensification, euphemisms, linguistic taboo, reversal of realities

\section{Povzetek}

\section{SARAJEVSKI VOJNI ARGO: PRIMER RAZMERJA MED JEZIKOM,} BESEDIŠČEM IN DOŽIVETIM

Dvajset let po koncu vojne se življenje v Sarajevu počasi postavlja v običajne tirnice. Spomini, pa naj bodo izraženi ali zamolčani, ostajajo. Pogled na vojno, ki se je odvijala skozi dolgotrajna obleganja, se je spremenil. Vprašanje pa je, kako. Težko je reči. Skupinski spomin nekatera dejanja še vedno (upravičeno ali neupravičeno) dojema in interpretira kot vojna, nekatera druga pa kot izdaje, saj rane še niso v celoti zaceljene.

Besedišče bosanskega argoja, ki se je uporabljal v vojni, pozna svoje evfemizme, tabuje, izogibanja, stalnice, ki pa se pomembno razlikujejo od vojnih argojev drugih jezikov, saj tri leta trajajoče obleganje ni enako vojni na fronti. V zadnji vojni v Bosni so bili »drugi« številni, tako sovražniki kot izdajalci. Razmere so torej idealne za razvoj argoja. V članku jih poskušamo osvetliti z naslonitvijo na zgodovinsko terensko raziskavo, ki je nujna za nadaljnjo pomensko analizo argojevskih leksemov, ki so nastali med vojno v Bosni.

Ključne besede: argo, obleganje Sarajeva, stopnjevanje, evfemizmi, jezikovni tabu, spreobračanje resničnosti 